\title{
Economic model predictive control of wastewater treatment plants based on BSM1 using linear prediction models
}

\author{
Rubén Moliner-Heredia ${ }^{1}$, Ignacio Peñarrocha-Alós ${ }^{1} \&$ Roberto Sanchis-Llopis ${ }^{1}$
}

\begin{abstract}
In this paper, we have developed an Economical Model Predictive Control (EMPC) for a Wastewater Treatment Plant (WWTP) with the use of a standard semidefinite programming solver. In this case, the objective has been to keep the ammonium concentration in the effluent under limits manipulating the air insufflation pumps at the biological reactor and an internal recycle valve. The minimized cost function consists of the product of the energy consumed by the air insufflator and the cost of the electricity, taking into account the variations of the tariffs over the day. We have simulated the behaviour of the WWTP using the Benchmark Model Simulation $\mathrm{n}^{0} 1$ (BSM1), and we have developed a linear prediction model in order to apply the EMPC method.
\end{abstract}

Keywords: BSM1, Wastewater Treatment Plants, Economic Model Predictive Control, Electricity Tariffs, Linear Prediction Models, Hammerstein-Wiener Models.

\section{INTRODUCTION}

Wastewater is one of the results of human activity. This polluted water must be treated before returning it to the environment. Wastewater Treatment Plants (WWTPs) are industrial facilities whose aim is to treat and cleanse wastewater in order to return it in acceptable conditions. Thus, WWTPs are crucial agents in an environment-friendly society, so an appropriate control of their behaviour is essential. Unfortunately, WWTPs are complex nonlinear dynamic systems subjected to large disturbances and uncertainties. This is due to the fact that these systems must face wide variations of the influent wastewater, and the biochemical and physical processes that happen in their inside feature limits and saturations. Therefore, controlling WWTP can be an intricate task. In the literature, different control strategies have been proposed. Most of those papers are based on the model that the Benchmark Model Simulation $\mathrm{n}^{\mathrm{o}} 1$ offers [1], which also has developed a control strategy that consists of two proportional-integral controllers (PI). Another paper [2], also takes into account this control method, and compares its performance with a Model Predictive Control (MPC) and with an Economic Model Predictive Control (EMPC) they have proposed. Other papers, such as refs [3]-[5], have also developed EMPC methods. While all of them use Performance Indexes (which are linear combinations of inner states of the WWTP utilized to check its performance assessment) or weighted variations of those indexes in order to minimize a cost function proposed by the BSM1, none of them take into consideration the electricity tariff variation,

\footnotetext{
${ }^{1}$ Departamento de Ingeniería de Sistemas Industriales y Diseño, Universitat Jaume I, Castellón de la Plana, Spain
}

which changes over the day. Besides, more control methods have been proposed, such as fuzzy control in [6]. A more deep dynamic analysis of the WWTPs is also addressed in [7]. On the other hand, some papers (ref [2]-[4]) apply the optimization (which is needed to solve the EMPC problem) directly onto the whole system, so it technically becomes a nonlinear model predictive control method. Actually, papers [3], [4] use a simplification of the BSM1 model, which reduces the calculation time (but it is still a nonlinear MPC). A linear prediction model would significantly reduce the calculation time while using the BSM1 equations, as well as the implementation cost.

\section{MODEL DESCRIPTION}

\section{A. Base Model}

In order to obtain an appropriate model of a WWTP, we have used the Benchmark Simulation Model $\mathrm{n}^{\circ} 1$ (BSM1) [1]. This model describes the behaviour of a biological reactor with two non-aerated compartments followed by three aerated compartments. The equations that regulate this reactor come from the Activated Sludge Model $\mathrm{n}^{\mathrm{o}} 1$ (ASM1). The BSM1 also describes the behaviour of the secondary clarifier. An explanation of these equations can be found in [8]. As it can be seen in Figure 1, influent wastewater $\left(Q_{i}\right)$ enters the bioreactor and crosses all the compartments. In the meanwhile, the bacteria stored in the reactor treats the wastewater, eliminating some components and generating some others. In the last compartment, there is a bifurcation, where some of the flow $\left(Q_{i n t}\right)$ is recycled back to the first compartment, and the other part flows to the secondary clarifier $\left(Q_{f}\right)$. Here, wastewater is subjected to a settling process, and the flow is yet divided into the effluent $\left(Q_{e}\right)$, which may be dumped directly into the river, and the underflow $\left(Q_{u}\right)$, which is rich in particulate components. This flow is partially purged to eliminate some of these particles, which results in the generation of sludge $\left(Q_{w}\right)$. The rest of the flow $\left(Q_{r}\right)$ is also recycled back to the first compartment of the biological reactor.

The differential equations that model the BSM1 use 13 different internal states for each compartment, which correspond with 12 components and a measure of alkalinity. In this paper, due to the recent improvements in the field of ammonium sensors, we have chosen the ammonia and ammonium concentration in the effluent flow $\left(S_{N H, e}\right)$ as the measured variable. In correspondence with the BSM1 example, the oxygen transfer coefficient in the fifth compartment $\left(k_{L} a_{5}\right)$ is a controllable input. Besides, we have considered 


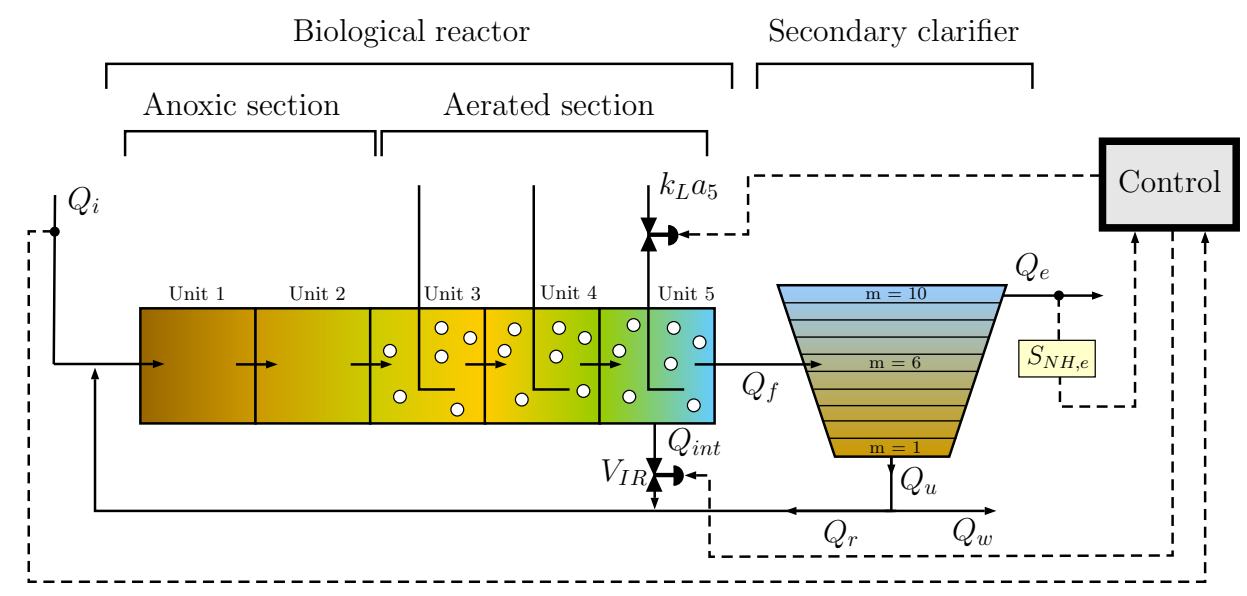

Fig. 1. BSM1 Model Adaptation.

that a valve $\left(V_{I R}\right)$ located in the internal recycle $\left(Q_{i n t}\right)$, which decides the percentage of the flow that returns back to the reactor, is also a controllable input.

\section{B. Modeling for prediction and control}

The BSM1 model defines the secondary treatment of a WWTP as a nonlinear system that can be described as a space-state model that requires up to 145 state variables. This is due to the substantial amount of inner processes that take place in each reactor:

$$
\begin{gathered}
\left\{\begin{array}{l}
\dot{x}(t)=f(x(t), u(t)), \\
y(t)=g(x(t)),
\end{array}\right. \\
x \in \mathbb{R}^{145} \quad u=\left[k_{L} a_{5}, V_{I R}\right] \in \mathbb{R}^{2} .
\end{gathered}
$$

In order to implement a predictive controller that can optimize the cost of the consumed energy with an acceptable computation time, the full nonlinear model (which is extremely complex) cannot be used in the optimization. Therefore, we need a simpler approximate model. The approach followed in this paper has been to use a linear discrete time model of a high order, completed with a nonlinear static term at its output to form a Wiener model (Fig. 2).

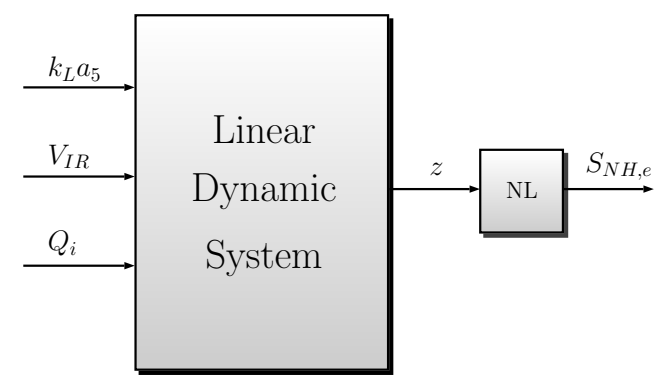

Fig. 2. Proposed Wiener Model for the plant.

The linear model consists of a difference equation of order $m$ relating the three considered inputs (control inputs $k_{L} a_{5}$ and $V_{I R}$ and disturbance $Q_{i}$ ), and an intermediate output signal $z$. The final output, $S_{N H, e}$, is a static nonlinear function of this intermediate variable $z$ :

$$
\left\{\begin{array}{l}
z_{k}=\sum_{j=1}^{j=m}\left(a_{j} z_{k-j}+b_{j} k_{L} a_{5, k-j}+\right. \\
\left.\quad+c_{j} V_{I R, k-j}+d_{j} Q_{i, k-j}\right)+K, \\
S_{N H, e}(k)=g\left(z_{k}\right) .
\end{array}\right.
$$

where the model coefficients $a_{j}, b_{j}, c_{j}, d_{j}$ and $K$ are calculated applying an identification procedure using data obtained from a simulation experiment performed in the nonlinear model. In this experiment, the input variables have been changed such that the input output data is sufficiently exciting for the frequencies of interest.

The nonlinear function $g(z)$ has been selected to be continuous, differentiable and monotonously increasing in the considered range, trying several candidates and choosing the one that minimizes the prediction error. Finally, function $g(z)=z^{2}$ has been selected as a reasonable compromise between simplicity and prediction accuracy.

Model (2) aims to approximate the behaviour of the nonlinear model (1) and is used in the prediction equations of the optimization algorithm. We assume that the inflow concentration is constant, as the primary treatment acts as a large capacity buffer tank, which filters out the variations of inflow concentrations.

\section{Electricity pricing}

In the Spanish electricity market, there are some special tariffs that are usually applied to industries which have high electricity consumption rates. These tariffs consist of several (three or six) pricing periods, distributed around the whole day, whose price does not depend on the market pool. In this paper, we take into account the daily oscillations of the electricity tariffs (ET). In this case, we assume that only the input variable $k_{L} a_{5}$ consumes electricity, as the consumption of valve $V_{I R}$ is negligible compared to it. To obtain the 
total cost of the electricity during a time span, we use this equation:

$$
E C_{5}=\frac{S_{O}^{s a t} \cdot V_{a s, 5}}{1.8 \cdot 1000} \int_{t=t_{0}}^{t=t_{f}} E T(t) k_{L} a_{5}(t) d t .
$$

where oxygen saturation concentration, $S_{O}^{s a t}$ and the volume of tank $5, V_{a s, 5}$ are parameters of the BSM1 model. This equation is an enhanced and modified version of the aeration energy AE from the BSM1 [1], as it includes the electricity cost variation, and only considers the energy consumption of the air insufflation pump in the fifth compartment, as only that one is assumed to be controlled. Calculating the cost of using the other air pumps is pointless, as they remain constant and no enhancement can be shown.

\section{Influent flow prediction}

An appropriate predictive control method requires a forecasting of the forthcoming disturbances. In the BSM1, the main disturbance is the influent flow, which, due to being the result of daily human activity, it roughly follows a certain daily and weekly pattern. However, the seasonal nature of human activity and natural random variations from day to day distort that pattern. Hence, the behaviour of the influent can be modelled as a sum of two components, as:

$$
Q_{i}(n)=h_{1}(n)+h_{2}(n),
$$

where $n$ is an index that defines the relative instant inside one week, $h_{1}$ represents the periodic predictable part, and $h_{2}$ represents the uncertain unpredictable part. The pattern $h_{1}$ is predicted by means of a lookup table that is filled with averaged measurements. As the weekdays patterns are different from the weekend ones, the table stores the values of each timestamp for a whole week. The table updating equation includes a first order averaging, so every sampling time the following equation is computed:

$$
\hat{h}_{1}(n)=p \hat{h}_{1}^{-}(n)+(1-p) Q_{i}(n) .
$$

where $\hat{h}_{1}^{-}(n)$ represents the value of the lookup table corresponding to instant $n$ of the week, which was updated the previous week. The online update of the table tries to maintain in a low value the part of the uncertainty $\left(h_{2}\right)$ that depends on the seasonal behaviour. In case of rainfall, the update of the table is simply interrupted, leaving the old values unchanged. On the other hand, the prediction of the influent flow is calculated as the value of the stored table $\left(\hat{h}_{1}(n)\right)$ for future instants, but as the real measured input flow $\left(Q_{i}(k)\right)$ for past instants. The past values are needed to compute the prediction model equations. Therefore, $\hat{Q}_{i}$ is calculated as:

$$
\begin{aligned}
& \hat{Q}_{i}\left(t_{0}+j\right)=\hat{h}_{1}\left(n_{0}+j\right), \\
& \hat{Q}_{i}\left(t_{0}-j\right)=Q_{i}\left(t_{0}-j\right) .
\end{aligned}
$$

where $n_{0}$ indicates the instant inside this week that corresponds to the absolute time $t_{0}$.

\section{DESCRIPTION OF THE PROPOSED CONTROL STRATEGIES}

The approach we have proposed is an Economic Model Predictive Control (EMPC) strategy. The fundamentals of EMPC are described in [9]. The main control objective is to minimize the cost of the electricity, expressed as a discretization of equation 3 , fulfilling some constraints regarding the range of the input signals and the limits in the output ammonia concentration:

$$
\min _{k_{L} a_{5}\left(t_{0}: t_{0}+H\right), V_{I R}\left(t_{0}: t_{0}+H\right)} J=\sum_{k=t_{0}}^{k=t_{0}+H} k_{L} a_{5}(k) E T(k),
$$

$$
\begin{array}{lr}
\text { s.t. } & \\
k_{L} a_{5}(k) \in\left[\underline{\left.k_{L} a_{5}, \overline{k_{L} a_{5}}\right]}\right. & k \in\left[t_{0}, t_{0}+H\right], \\
V_{I R}(k) \in\left[\underline{V_{I R}}, \overline{V_{I R}}\right] & k \in\left[t_{0}, t_{0}+H\right], \\
\hat{z}_{k}=g^{-1}\left(\overline{S_{N H, e, k}}\right) & k \in\left[t_{0}-m, t_{0}\right], \\
\hat{z}_{k} \in\left[g^{-1}\left(\underline{S_{N H, e}}\right), g^{-1}\left(\overline{S_{N H, e}}\right)\right] & k \in\left[t_{0}, t_{0}+H\right], \\
\hat{z}_{k}=\sum_{j=1}^{j=m}\left(\underline{a_{j} \hat{z}_{k-j}+b_{j} k_{L} a_{5, k-j}+}\right. & k \in\left[t_{0}+1, t_{0}+H\right] . \\
\left.\quad+c_{j} V_{I R, k-j}+d_{j} \hat{Q}_{i, k-j}\right)+K &
\end{array}
$$

The control objective consists of the minimization of the total electricity cost of the aeration during a prediction horizon $H$ (integral along the period of the product of the electric power resulting from $k_{L} a_{5}$ times the electricity cost $E T(t))$. The main constraint to be fulfilled is the allowed upper limit of ammonium concentration in the effluent $\left(\overline{S_{N H, e}}\right)$. The future ammonium evolution is predicted using the approximate model (2). On the other hand, $k_{L} a_{5}$ and $V_{I R}$ are also restricted to the actuator limits $\left(\overline{k_{L} a_{5}}, \underline{k_{L} a_{5}}, \overline{V_{I R}}\right.$, $\left.\underline{V_{I R}}\right)$.

This optimization problem can be unfeasible in some instants. This could happen due to uncertainties caused by approximating the whole nonlinear model with a Wiener model, or due to miscalculations of the influent flow prediction. If this happens, a secondary tracking optimization controller is ready to obtain a suitable control action:

$$
\min _{k_{L} a_{5}\left(t_{0}\right), V_{I R}\left(t_{0}\right)} J=\left(\overline{S_{N H, e}}-g\left(\hat{z}_{t_{0}+1}\right)\right)^{2}
$$

$$
\begin{aligned}
& \text { s.t. } \\
& k_{L} a_{5}\left(t_{0}\right) \in\left[k_{L} a_{5}, \overline{k_{L} a_{5}}\right], \\
& V_{I R}\left(t_{0}\right) \in\left[\overline{\left.V_{I R}, \overline{V_{I R}}\right],}\right. \\
& \hat{z}_{k}=g^{-1}\left(\overline{S_{N H, e}, k}\right) \\
& \hat{z}_{k}=\sum_{j=m}^{j=1}\left(a_{j} \hat{z}_{k-j}+b_{j} k_{L} a_{5, k-j}+,\right. \\
& \left.\quad+c_{j} V_{I R, k-j}+d_{j} \hat{Q}_{i, k-j}\right)+K
\end{aligned}
$$

This secondary controller (TMPC) tries to minimize the difference between the ammonia output concentration and 
the upper limit. We use the same prediction model in order to predict the future value of $z_{k}$ and therefore, the value of $S_{N H, e}$.

Every sampling time the EMPC optimization is run first. If it delivers a feasible solution, it is applied until next period. However, if no feasible solution is obtained from EMPC, the TMPC optimization is run, and the obtained solution applied until next sampling time.

\section{Simulation Results}

\section{A. Simulation settings}

In this subsection, we explain the parameters that have been used to implement the EMPC.

The influent data is provided by the BSM1 [1]. In this case, we have used the dry weather data set repeatedly throughout the length of the simulation. As we have only used dry weather data, the rain effect is not taken into account.

The volumes of the compartments at the biological reactor are also provided by the BSM1. They are $V_{a s, 1}=V_{a s, 2}=$ $1000 \mathrm{~m}^{3}$ and $V_{a s, 3}=V_{a s, 4}=V_{a s, 5}=1333 \mathrm{~m}^{3}$.

The electricity tariff we have chosen corresponds to working winter days for Spanish tariff 3.1A., leading to an electricity price, $E T(k)$ that varies along the day as shown in figure 5. The reason is because in this tariff there are a lot of variations of the price over the day, so the performance of this EMPC method is tested in an unfavorable case.

We have used the toolbox YALMIP to prepare the optimizer sedumi, which minimizes the objective function.

The air insufflators in compartments 3 and 4 of the biological reactor are working at a constant rate of $k_{L} a_{3}=$ $k_{L} a_{4}=240 d^{-1}$, while compartments 1 and 2 are anoxic, so $k_{L} a_{1}=k_{L} a_{2}=0 d^{-1}$. The air insufflator in compartment 5 , as the main controllable input, can work in a range of $k_{L} a_{5} \in[0,240] d^{-1}$.

On the other hand, the signal $V_{I R}$ is defined as the percentage of flow from compartment 5 that gets recycled back $\left(Q_{\text {int }}\right)$ to the first compartment, that is changed using a valve. Therefore, it ranges from $10 \%$ to $90 \%$ to prevent completely closed loops or bypasses $\left(V_{I R} \in[0.1,0.9]\right)$. Besides, in order to avoid undesired effects of sudden changes of flow in valves, we have added a small time filter to smooth the changes of the variable $V_{I R}$.

We have set the ammonium limit $\overline{S_{N H, e}}$ to $9 \mathrm{gN} \cdot \mathrm{m}^{-3}$, while setting $S_{N H, e}$ to 0 to indicate mathematically the lower real limit of a concentration. The legal requirement for the upper limit refers to a daily average below 9. However, we have set it as an instantaneous limit, to cope with transient violations due to the approximate prediction model and disturbance uncertainties.

We have performed the simulation of the BSM1 WWTP dynamic model using a time sampling of $1 \mathrm{~min}$. However, we executed the control algorithm with a higher sampling period of $60 \mathrm{~min}$. We have chosen an order $m=13$ for the linear dynamic model of the WWTP used in the predictor, i.e., the predictor model uses the last $m=13$ hours of data. The prediction horizon of the predictive control is $H=$
$32 \mathrm{~h}$. The experiment simulates 39.66 days of control and operation.

With the aim of a comparative analysis of the behaviour of the proposed EMPC, we considered a simple relay-based control as an alternative. We chose that method because it is a common, simple and effective way to control the ammonium concentration. This control method only activates the air pump in compartment 5 when the ammonium effluent surpasses the threshold $\overline{S_{N H, e}}$. The logic of the relay is shown here:

$$
\left\{\begin{array}{llr}
\text { if } S_{N H, e} \geq \overline{S_{N H, e}}, & k_{L} a_{5}= & 240 d^{-1} \\
\text { if } S_{N H, e}<\overline{S_{N H, e}}, & k_{L} a_{5}= & 0 d^{-1}
\end{array} .\right.
$$

The valve $V_{I R}$ remains in this case static at $60 \%$, which recycles the flow in the same proportion as the stable conditions from BSM1. As for $k_{L} a_{x}$ in compartments 1 to 4 , the conditions are identical to the previous case, as $k_{L} a_{1}=k_{L} a_{2}=0 d^{-1}$ and $k_{L} a_{3}=k_{L} a_{4}=240 d^{-1}$.

\section{B. Simulation results}

In this subsection, we show and discuss the simulation results.

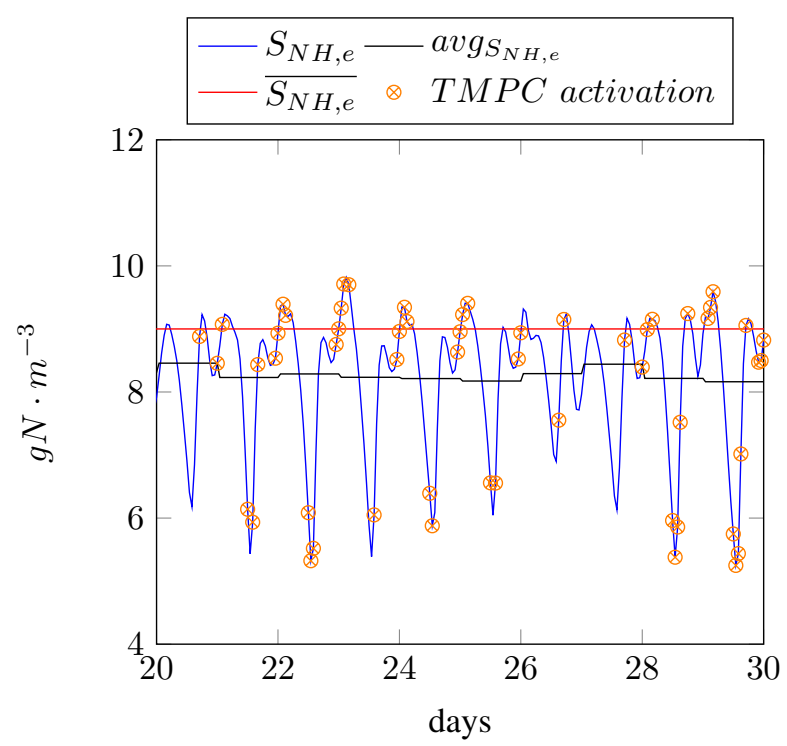

Fig. 3. $S_{N H, e}$ under limits using the EMPC method.

1) Keeping $S_{N H, e}$ under limits: Figure 3 shows the values of $S_{N H, e}$ from day 20 to day 30 . We took this sample to facilitate comprehension. As it can be seen, most of the time the effluent ammonium concentration remains under the limit $\left(\overline{S_{N H, e}}\right)$. There is an explanation for the moments that $S_{N H, e}$ surpasses the upper limit. This happens because the Wiener model is an approximation of the nonlinear system, and sudden signal peaks may not always be detected by the EMPC, who interprets them as still being under limits. The uncertainty of the inlet flow prediction is another factor that makes the final ammonium surpass the limit. However, the average daily value of $S_{N H, e}$ (expressed as $a v g_{S_{N H, e}}$ ) shows 


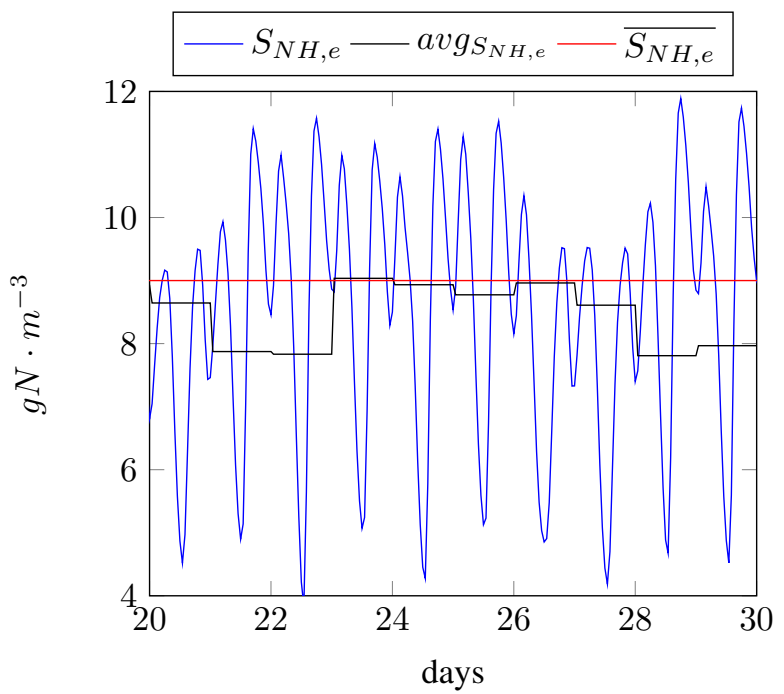

Fig. 4. $S_{N H, e}$ under limits using the relay-based control method.

that it always remains well under the limits, which is the desired result.

On the other hand, Figure 4, shows that the ammonium output $S_{N H, e}$ surpasses the limit far more frequently and with more intensity with the relay control than with the EMPC method. The average $a v g_{S_{N H, e}}$ also fluctuates more, crossing the limits in some occasions, and in others the average goes under $8 \mathrm{~g} \mathrm{~N} \cdot \mathrm{m}^{-3}$. This shows that the EMPC optimizes the consumed energy, as it keeps $S_{N H, e}$ under limits without overusing the air pump.

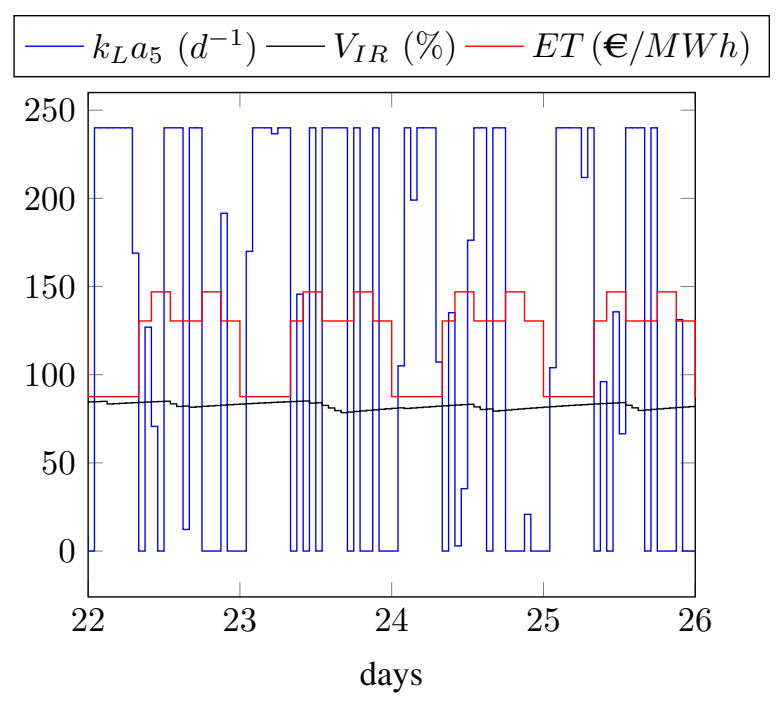

Fig. 5. Electricity tariff function and behaviour of the inputs (EMPC method).

2) Effect of the tariffs on the actuators: Figure 5 shows the actuator and the electricity tariff values, so the effectiveness of the EMPC method can be displayed. The ET changes along the day. The graph shows that in the cheapest periods $k_{L} a_{5}$ works at its maximum power, trying to minimize the $S_{N H, e}$ output at minimum cost. On the other hand, note that

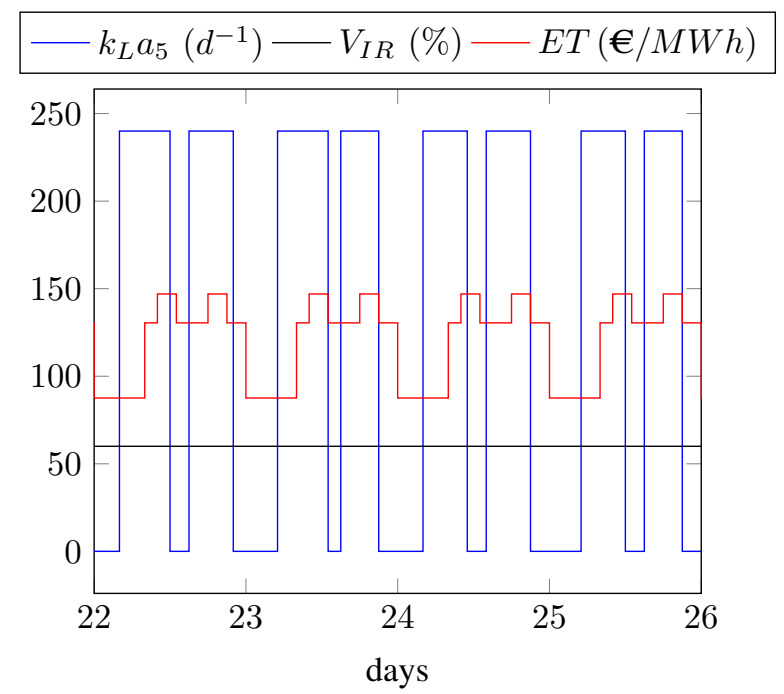

Fig. 6. Electricity tariff function and behaviour of the inputs (relay method).

in the more expensive periods, $k_{L} a_{5}$ is off or in lower values, and only activates if it has no choice to keep $S_{N H, e}$ under limits. The valve $V_{I R}$ is gradually opened (recycling more flow) in the cheapest periods, increasing $S_{N H, e}$, so in the most critical and expensive periods it can be closed, reducing $S_{N H, e}$ and the need of aeration.

On the other hand, Figure 6 shows that, as expected, the relay control method activates and deactivates the air pump $k_{L} a_{5}$ without taking into account the electricity cost. The valve $V_{I R}$, as we have explained before, remains fixed at $60 \%$.

3) Cost comparison of the implemented methods: Figure 7 shows the daily cost of aeration of tank 5 (in $€$ ). Both control methods can be compared. We have obtained the values using equation 3 . It can be seen that the daily cost with the EMPC method is clearly lower than the cost with the relay control method. The cost reduction is between $20 \%$ and $30 \%$ depending on the day. This could represent, for a small plant, around $10000 €$ in savings per year. And this savings are obtained while at the same time the ammonium concentration control is improved, as we explained. The weekly variations can also be observed in this plot; the cost in the weekends is lower because the input flow is lower in the weekends, according to the BSM1 data set used.

\section{CONCLUSIONS}

In this paper, we have presented an Economic Model Predictive Control for the regulation of a wastewater treatment plant. The objective is the minimization of the energy cost of the aeration process, taking into account the electricity tariffs, which vary along the day. The output variable that is assumed to be measured is the ammonium concentration at the outlet flow. This variable must be maintained below a given threshold defined by legal requirements. The main control action is the aeration of the last compartment of the plant, which is assumed to be performed by an electrical air pump. 


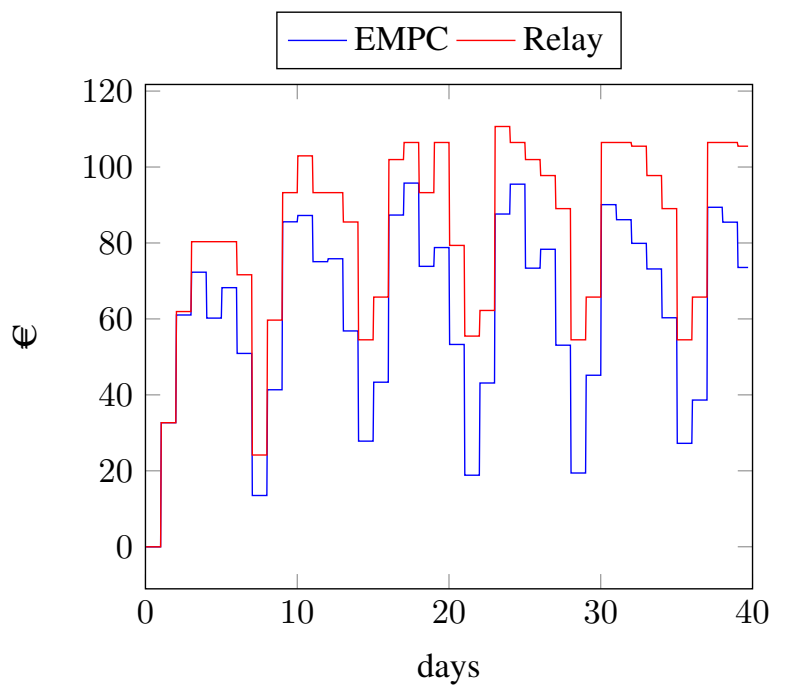

Fig. 7. Comparison of the total cost of aerating compartment 5 using each control methods.

We have used the BSM1 model in order to simulate the plant behaviour. This is a highly nonlinear complex model with 145 states. In order to define a low computational cost optimization problem for the predictive controller, we have proposed a simpler model of the plant. It consists of a linear discrete time model with a nonlinear static function at the output. We have obtained the parameters of this model by identification from the simulated nonlinear plant.

We also defined a simple input flow predictor. It consists of a one week length lookup table that is updated online with every input flow measurement using an updating filter.

We carried out some simulations to test the validity of the approach. The behaviour with respect the ammonium concentration at the outlet flow is correct. The concentration surpasses the upper limit sporadically, due to uncertainties in the input flow prediction and due to the approximate model used, but the average daily concentration is clearly below the limit. On the other hand, as we expected, the controller tries to use maximum aeration during the cheapest tariff periods, while trying to reduce the aeration during the most expensive ones. The result is a significant overall cost reduction if compared, for example, with a simple relay controller.

\section{ACKNOWLEDGMENTS}

This work has been supported by MICINN project number TEC2015-69155-R from the Spanish government and the grant ACIF/2018/245 from Generalitat Valenciana.

\section{REFERENCES}

[1] ALEX, J., et al. Benchmark simulation model no. 1 (BSM1). Report by the IWA Taskgroup on Benchmarking of Control Strategies for WWTPs, 2008.

[2] ZENG, Jing; LIU, Jinfeng. Economic model predictive control of wastewater treatment processes. Industrial \& Engineering Chemistry Research, 2015, 54.21: 5710-5721.
[3] REVOLLAR, S., et al. Optimization of economic and environmental objectives in a Non Linear Model Predictive Control applied to a wastewater treatment plant. In: System Theory, Control and Computing (ICSTCC), 2016 20th International Conference on. IEEE, 2016. p. 318-323.

[4] REVOLLAR, S.; VEGA, P.; VILANOVA, R. Economic optimization of wastewater treatment plants using Non Linear model predictive control. In: System Theory, Control and Computing (ICSTCC), 2015 19th International Conference on. IEEE, 2015. p. 583-588.

[5] REVOLLAR, Silvana, et al. Optimal Control of Wastewater Treatment Plants Using Economic-Oriented Model Predictive Dynamic Strategies. Applied Sciences, 2017, 7.8: 813.

[6] SANTÍN, I.; PEDRET, C.; VILANOVA, R. Fuzzy control and model predictive control configurations for effluent violations removal in wastewater treatment plants. Industrial \& Engineering Chemistry Research, 2015, 54.10: 2763-2775.

[7] FRANCISCO, Mario; SKOGESTAD, Sigurd; VEGA, Pastora. Model predictive control for the self-optimized operation in wastewater treatment plants: Analysis of dynamic issues. Computers \& Chemical Engineering, 2015, 82: 259-272.

[8] VILANOVA, Ramon; SANTÍN, Ignacio; PEDRET, Carles. Control y operación de estaciones depuradoras de aguas residuales: Modelado y simulación. Revista Iberoamericana de Automática e Informática Industrial RIAI, 2017, 14.3: 217-233.

[9] RAWLINGS, J.; ANGELI, D.; and BATES, C. (2012). Fundamentals of Economic Model Predictive Control. In Annual Conference on Decision and Control (CDC).IEEE, volume 10, pages 3851-3861. 2012. 